\title{
Endoscopic Decompression, Foraminalplasty and Dorsal Rhizotomy for Foraminal Stenosis and Lumbar Spondylosis: A Hybrid Procedure in lieu of Fusion
}

\author{
Anthony T. Yeung ${ }^{1,2^{*}}$ \\ ${ }^{1}$ University of New Mexico School of Medicine, Albuquerque, New Mexico \\ ${ }^{2}$ Desert Institute for Spine Care, Phoenix, Arizona, USA \\ "Corresponding author: Anthony T. Yeung, Desert Institute for Spine Care, Phoenix, Arizona, USA, Tel: +1 602-216-6082; E-mail: ayeung@sciatica.com
}

Received date: November 15, 2016; Accepted date: December 09, 2016; Published date: December 16, 2016

Copyright: @ 2016 Yeung AT, et al. This is an open-access article distributed under the terms of the Creative Commons Attribution License, which permits unrestricted use, distribution, and reproduction in any medium, provided the original author and source are credited.

\begin{abstract}
Surgical Treatment of chronic Low Back pain syndrome from an aging spine traditionally focuses on fusion as the ultimate surgical means to eliminate debilitating back pain and sciatica." A variety of painful degenerative conditions of the lumbar spine is treated surgically by translaminar decompression and fusion. Spine surgeons trained to perform fusions, with no training in endoscopic surgery are battling an economic crisis of escalating cost and affordability when fusion surgery is recommended for chronic back pain. This is considered the ultimate treatment by surgeons favoring fusion as a means to treat perceived or actual instability and/or deformity supported by imaging studies.
\end{abstract}

Keywords: Dorsal Endoscopic Rhizotomy; Endoscopic Foraminoplasty; Dorsal Ramus; Foraminal Stenosis; Lumbar Spondylosis; Facet Arthrosis; Hybrid Procedure

\section{Introduction}

Instrumented fusion favored by Herkowitz, is currently being challenged by recent publications in 2016. Aging, however, can progress to stenosis, degenerative instability and deformity that are dependent on individual anatomic and physiologic differences. Disc degeneration, also affected by individual and facet sagittal alignment affects each person's response to normal progression of their aging process.

Comparative cost effectiveness QUALY studies, the new recent focus, supporting fusion, may have level 1 or 2 Evidence Based outcomes to support each procedure studied. When surgical instrumentation and implant costs are factored in, and when instrumented fusion is considered the "gold standard", It is still considered economically unsustainable by payers due to the added cost of hardware and implants to achieve pain relief. Denials for surgical pre-operative authorization and payment are therefore becoming more and more frequent, changing the dynamics of affordable healthcare.

Continuous radiofrequency (CRF) and pulsed radiofrequency (PRF) lesioning of the medial branch of the dorsal ramus, an interventional surgical technique to treat facet mediated axial back pain, is a current method utilized by pain management physicians for the treatment of chronic axial lumbar back pain. Clinically, however, even when "successful", relief with fluoroscopically guided radiofrequency lesioning may be only temporary, effective about $50 \%$, and short lived.

Armed with surgical experience obtained from endoscopic foraminal surgery and visualized dorsal rhizotomy, better and more lasting results can be obtained. This is due to a better understanding of the pathogenesis of low back pain, aided by new information obtained by meticulous fresh cadaver dissection demonstrating variable anatomy. A dorsal and foraminal Endoscopic visualized decompression and rhizotomy technique was developed to effectively treat common and chronic low back pain as a hybrid procedure.

An IRB approved procedure by Saint Luke's Medical Center, Phoenix Arizona, to study endoscopically visualized patho-anatomy and its relation to pain generators was used for this study on the viability of transforaminal endoscopic decompression of painful degenerative conditions of the lumbar spine. The endoscopic system by Richard Wolf is FDA $510 \mathrm{k}$ approved as the Yeung endoscopic spine system was followed by a specialized Endoscopic Rhizotomy System.

A pilot study was started n 2005 on endoscopically guided dorsal rhizotomy, that has continued since the first 50 consecutive patients, picked as a pilot study when the pilot study reached 50 patients with at least 1-2 years follow-up. The demonstrating successful decrease in common and chronic low back pain, was initially reported at the IMISS meeting January 2006 in Zurich. Since 2005, over 1,000 procedures have now been performed with the same outcome.

A separate unpublished study on transforaminal decompression of foraminal stenosis from the author's database demonstrated similar success of endoscopic foraminal stenosis for sciatica and intermittent claudication also produced relief of axial back pain. With patient's exhibiting similar percentages of back pain and sciatica, combining both procedures as a hybrid procedure provided both relief of sciatica, intermittent claudication and back pain that demonstrated reduction of common back pain following endoscopically guided foraminoplasty.

\section{Methods}

Cadaver studies of the anatomy of the dorsal ramus further determined that, while the medial branch to the facet joint usually crosses the transverse process, there were variations of the anatomy of the dorsal ramus and its branches. Because this study was a cadaver study on the anatomy of the dorsal ramus, no IRB approval was 
Citation: Yeung AT (2016) Endoscopic Decompression, Foraminalplasty and Dorsal Rhizotomy for Foraminal Stenosis and Lumbar Spondylosis: A Hybrid Procedure in lieu of Fusion. J Neurol Disord 4: 322. doi:10.4172/2329-6895.1000322

Page 2 of 4

deemed needed for this study, but IRB approval was obtained from St. Lukes Medical Center's IRB committee to study the role of transforaminal endoscopic decompression of the lumbar spine when the Yeung endoscopic Spine system was approved by the FDA. The dorsal ramus is a sensory nerve that originates in the foramen ventral to the intertransverse ligament, It exits dorsally to send branches to the facet joint and the paravertebral musculature. The medial branch can be ablated in the foramen (Figures 1 and 2) or, more consistently, as it crosses the transverse process before innervating the facet joint $[1,2]$. These cadaver studies demonstrated significant anatomic variations that supported the need for visualized rhizotomy.

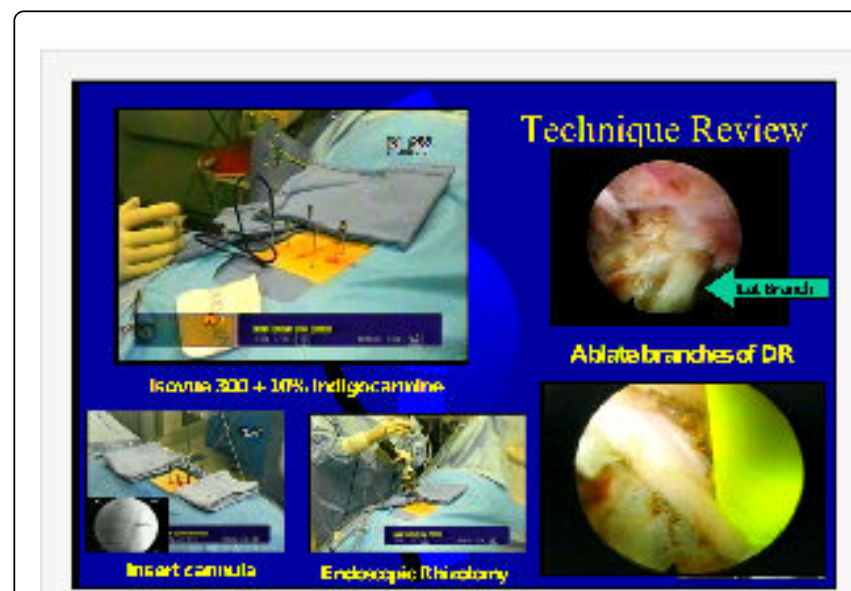

Figure 1: The visualized dorsal endoscopic surgical approach with the Endoscopic Rhizotomy system by Richard Wolf. The Endoscopic visualized Dorsal Rhizotomy Procedure. Visualizing and ablating the nerves crossing the transverse process to innervate the facet joint. The use of indigocarmine at the transverse can help identify communication with the foramen and explain why the procedure can help sciatica. The branches of the dorsal ramus do not have to be visualized to get good results from visualized ablation of the nerves, shown in the images above.

Dorsal Ramus Rhizolysis was studied in a pilot study of 50 patients arbitrarily determined in 2005 since the last of 50 consecutive patients had over 1 year follow-up. (1) A retrospective non randomized study of the 50 initial patients was then used to assess the efficacy of endoscopic visualized rhizotomy for the relief of chronic common low back pain. Patients with lumbar spondylosis and facet arthrosis who had at least $50 \%-70 \%$ pain relief by medial branch blocks met the inclusion criteria for the visualized, surgically directed endoscopic technique. A specially designed cannula and endoscope (Richard Wolf, GmBh) was developed specifically to facilitate this surgery. The surgical technique refinement was guided by anatomic observations from additional cadaver dissections and endoscopic visualization of foraminal nerves that revealed variable locations of the medial branch. Since 2005, approximately 1,000 patients have undergone endoscopic visualized dorsal rhizotomy [2-4].

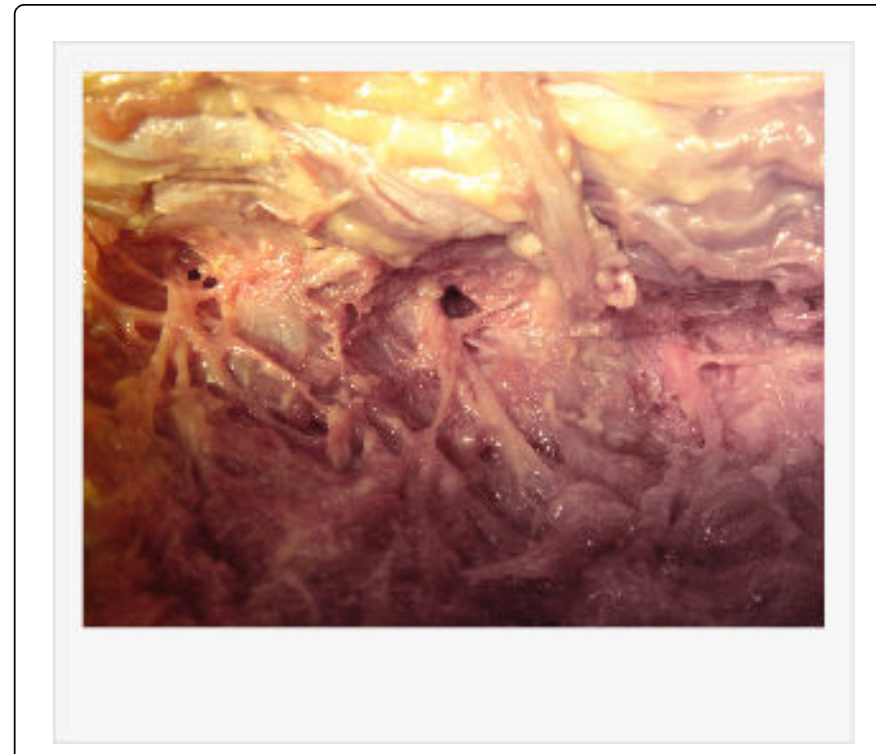

Figure 2: Cadaver dissection of the branches of the Dorsal Ramus. These branches of the dorsal ramus can be visualized ventral to the intertransverse ligament or on the transverse process. Foraminal decompression can therefore provide back pain relief and well as dorsal rhizotomy from ablation and resection of sensory nerves innervation the facet joints. The endoscopic technique provides more lasting and predictable results than continuous or pulsed radiofrequency utilized by pain management physicians. Dissection by Yinggang Zheng, M.D. YESS fellow.

\section{Results}

Average pre-operative VAS, ODI and Oswestry scores as well as patient satisfaction, all demonstrate significant reduction of preoperative back pain. Vas score decreased from 6.2 to 2.5. Oswestry score decreased from $48-28$.

\section{No patient was worse}

Improvement remained with about 10 percent regression in a 10 year follow review from a personal electronic database from 2005 to 2016. This compared favorably with published and historical statistical database for more invasive surgical treatment of common and chronic low back pain. Endoscopic foraminoplasty allowed for endoscopic visual stimulation of pain, and elimination of pain following foraminal decompression of the pain generators in the disc and foramen in an awake patient that combines disc decompression, foraminoplasty, and electro thermal and laser closure of "toxic" annular tears [2-5].

The traversing and exiting nerve in the axilla forming the "hidden zone" of Mac Nab is specifically targeted for decompression. When axial back pain is significant, dorsal rhizotomy is added as a hybrid procedure. The surgical procedure is documented in an awake patient under only local anesthesia with minimal sedation serving as "immediate" surgically, and clinically based evidence.

An endoscopic transforaminal decompressive technique that does not contribute to spinal instability has also evolved into a reliable, and effective technique for surgically addressing chronic back pain. (Figure 3) This ablation procedure with endoscopic assist, was found to be an 
Citation: Yeung AT (2016) Endoscopic Decompression, Foraminalplasty and Dorsal Rhizotomy for Foraminal Stenosis and Lumbar Spondylosis: A Hybrid Procedure in lieu of Fusion. J Neurol Disord 4: 322. doi:10.4172/2329-6895.1000322

Page 3 of 4

effective alternative to early fusion, especially when the endoscopic foraminoplasty technique was combined with dorsal rhizotomy. If staged, reserving fusion as a "salvage" procedure, about $75 \%$ of patients were spared a later fusion procedure, especially in elderly patients over 70 years old. The procedure incorporates intradiscal and foraminal decompression, targeting nerves that innervate the disc and posterior column through endoscopic rhizolysis.

\section{Discussion}

Because of the excellent results from the pilot study, the second phase of the study evolved from visualizing additional nerves during endoscopic foraminal decompression. It was discovered that foraminoplasty also provided back pain relief.

This resulted in further reviewing cadaver anatomy and recognized by transforaminal decompression of the lateral recess. Patient selection expanded to "tweaking" the initial inclusion criteria to combine foraminoplasty for foraminal stenosis combined with dorsal visualized rhizotomy in elderly patients with stenosis and lumbar spondylosis. This provided even more consistent and additional patient satisfaction with consistently predictable good/excellent patient satisfaction ratings. Probing under local anesthesia, we were also able to determine whether small non-painful nerves could be safely ablated, but if the furcal nerve elicited pain or was clearly a branch from a spinal nerve such as the exiting nerve, it is best to avoid cutting or ablating the nerve (Figures 3 and 4 ).

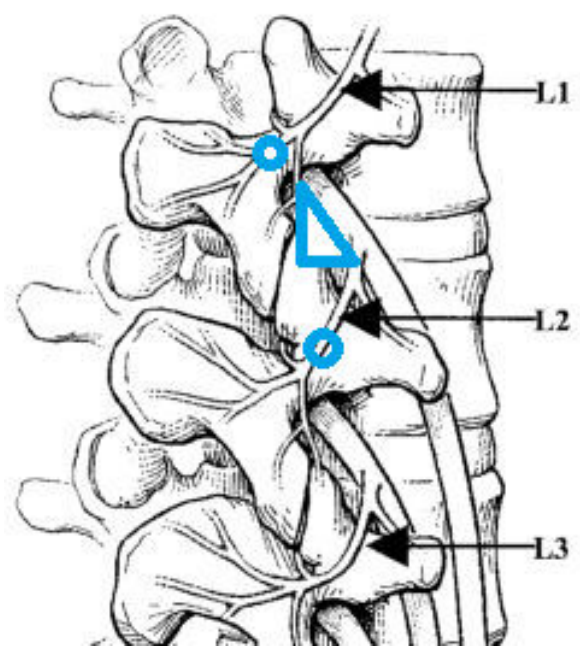

Figure 3: Schematic drawing of Kambin's Triangle demonstrating where the medial branch of the dorsal ramus can be visualized in the foramen endoscopically ventral to the facet and as it crosses the transverse process. It is difficult to differentiate a furcal nerve that is a branch of a mixed sensory and motor nerve as opposed to a pure sensory nerve from the dorsal ramus.

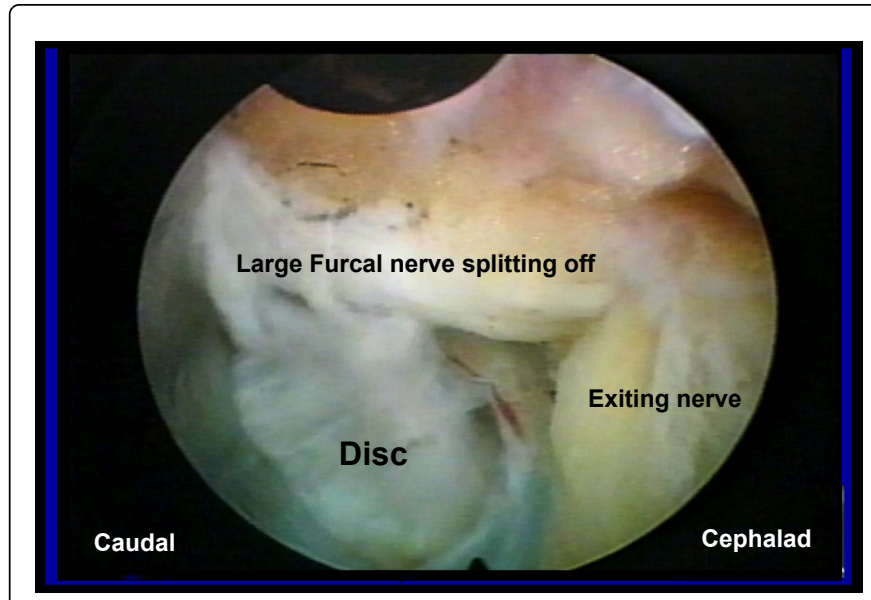

Figure 4: Endoscopic Foraminal view of a furcal nerve arising from the exiting nerve.

It is difficult to differentiate these sensory nerves arising from the dorsal ramus from peripheral nerves that were branches of the normal spinal nerve labled "furcal nerves". Furcal nerves can eminate from mixed motor or sensory nerves. Spinal nerves usually produce pain when stimulated by probing or by RF stimulation. The patient is able to respond when the patient is awake and only lightly sedated. Therefore, minor variations and modifications of the surgical technique was incorporated based on visualization and ablation of foraminal nerves recognized to be from the dorsal ramus and it's branches or a furcal nerve Figures $5 a$ and $5 b$ ).

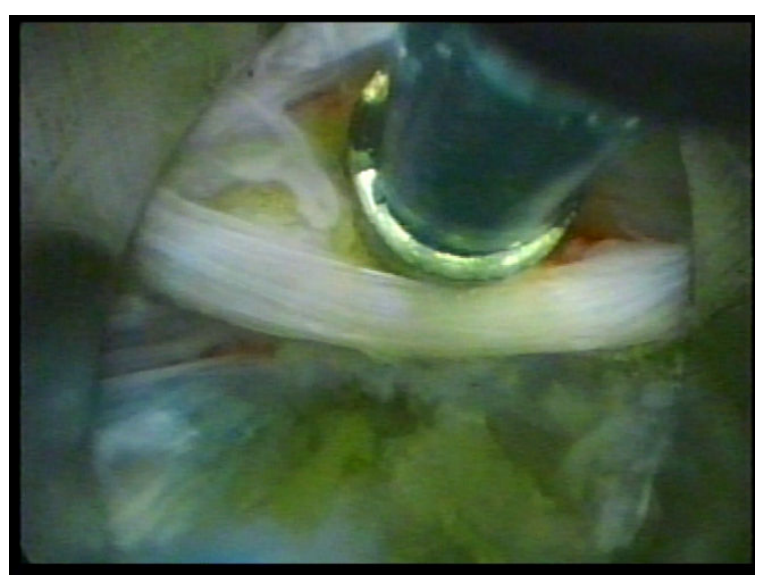

Figure 5a: Foraminal view of a branch of the dorsal ramus nerve in the foramen that was ablated or cut because it did not cause pain when probed or stimulated with Radiofrequency. Judgment and experience will provide information to the surgeon in trying to differentiate furcal nerves versus branches of the dorsal ramus. 
Citation: Yeung AT (2016) Endoscopic Decompression, Foraminalplasty and Dorsal Rhizotomy for Foraminal Stenosis and Lumbar Spondylosis: A Hybrid Procedure in lieu of Fusion. J Neurol Disord 4: 322. doi:10.4172/2329-6895.1000322

Page 4 of 4

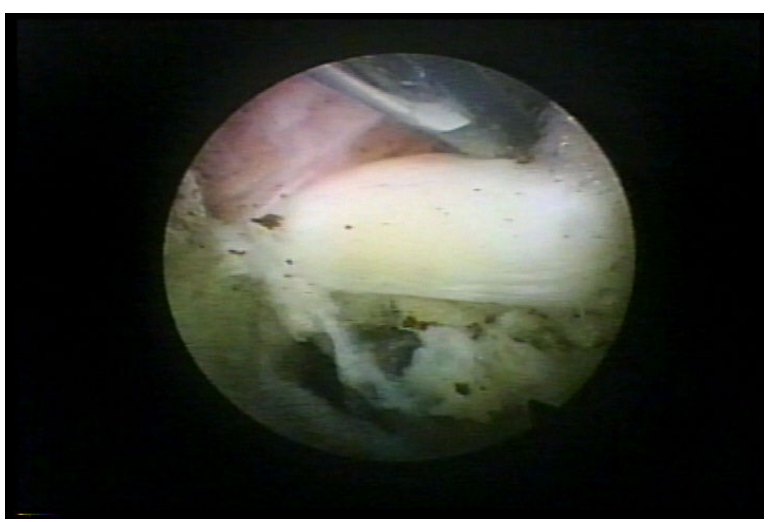

Figure 5b: Large nerves in the foramen are difficult to differentiate a branch of the Dorsal Ramus, a SENSORY nerve, from a furcal nerve branch from a spinal nerve that has sensory as well as motor fibers. If the nerve is large $(2-3 \mathrm{~mm})$ It is best to avoid cutting or ablating it, especially if pain is evoked by RF stimulation.

We have also even seen neuromas in the foramen. Decompressing the neuroma, whether cut or freed can also give back pain relief. We now also look for nerve branches in the foramen during transforaminal disc decompression and foraminoplasty. We can safely ablate small nerves of $1 \mathrm{~mm}$ or less. This allowed more meticulous identification and ablation of the branches off the dorsal ramus as well as the intermediate and lateral branches of the dorsal ramus, including releasing the inter transverse ligament and membrane to decompress the Dorsal Ramus.

Because of this observation, carefully selected patients with severe deformity and degenerative scoliosis who are high risk candidates for surgical correction due to age, increased surgical morbidity, or more risk for fusion, is a candidate for the hybrid procedure. We can even include patients with adjacent level discogenic and non-discogenic axial back pain, and carefully selected workman's comp patients,

There have been no patients in the disappointed, and not satisfied group when $70 \%$ or greater relief diagnostic and therapeutic injections with transforaminal epidural blocks and with medial branch blocks was used for surgical inclusion criteria. This is attributed to more accurate patient estimation of pain relief following injections performed by the surgeon. As surgical experience, patient selection refinement, and experience with the endoscopic technique evolved, This author is able to warrantee a favorable result from shared decision to try the recommended ttansforaminal procedure, first, or an alternative more traditional open procedure can be considered, such as fusion.
This technique has evolved and culminated in predictable clinical results, supported by results predicted by diagnostic and therapeutic injections [2-4]. Surgical decompression creates minimal surgical morbidity when operations are by highly experienced endoscopic surgeons. This clinical observation is supported by peer-reviewed publication. No publication have refuted the documented results $[5,6]$. Modifications of the surgical technique over the years is aided by improved instrumentation endoscopic translaminar and transforaminal surgical techniques so that a "full endoscopic technique or combined surgical technique is possible.

Level I evidence for endoscopic spine surgery under local is difficult and not easily validated for blinded comparison, so other evidence such as direct visualization of the surgical treatment of the pain generator and the clinical results of rhizolysis may need to be substituted for traditional evidence based scientific validation.

\section{Conclusion}

Hybrid endoscopic transforaminal decompression, combined with dorsal rhizotomy will open the door for early cost effective surgical solutions for painful degenerative conditions of the lumbar spine in lieu of fusion, this hybrid technique warrants further study and adoption by surgeons trained adequately in this technique.

\section{References}

1. Yeung A (2007) Endoscopic medial branch and dorsal ramus rhizotomy for chronic Axial back pain: a Pilot Study. International 25th Jubilee Course on Percutaneus Endoscopic Spine surgery and Complementary Techniques. Zurich Switzerland January 24-25.

2. Yeung AT, Yeung CA, Zheng Y (2010) Dorsal Endoscopic Rhizotomy for Chronic Nondiscogenic Axial Low Back Pain in The Comprehensive Treatment of the Aging Spine: Minimally Invasive and Advanced Techniques. Editors Yue Guyer Johnson Khoo.

3. Yeung AT, Gore S (2011) In-vivo Endoscopic Visualization of Pathoanatomy in Symptomatic Degenerative Conditions of the Lumbar Spine II: Intradiscal, Foraminal, and Central Canal Decompression. Surg Technol Int 299-319.

4. Yeung AT, Gore S Endoscopically Guided Foraminal and Dorsal Rhizotomy for Chronic Axial Back Pain Based on Cadaver and Endoscopically Visualized Anatomic Study. Int J Spine Surg 8: 23.

5. Gore S, Yeung AT (2014) The "inside out" transforaminal technique to treat lumbar spinal pain in an awake and aware patient under local anesthesia: results and a review of the literature. Int J Spine Surg.

6. Li ZZ, Hou SX, Shang WL, Song KR, Wu WW (2014) Evaluation of endoscopic dorsal ramus rhizotomy in managing facetogenic chronic low back pain. Clinical Neurology and Neurosurgery 126: 11-17. 